\title{
Physical interpretation of the NGC 7469 UV/X-ray variability
}

\author{
P. O. Petrucci ${ }^{1}$, L. Maraschi ${ }^{2}$, F. Haardt ${ }^{3}$, and K. Nandra ${ }^{4}$ \\ ${ }^{1}$ Laboratoire d'Astrophysique de Grenoble, BP 43, 38041 Grenoble Cedex 9, France \\ 2 Osservatorio Astronomico di Brera, via Brera 28, 20121 Milano, Italy \\ e-mail: maraschi@brera.mi.astro.it \\ 3 Dipartimento di Scienze, Universitá dell'Insubria, via Lucini 11, 22100 Como, Italy \\ e-mail: Francesco.Haardt@mib.infn.it \\ 4 Astrophysics group, Imperial College London, Blackett Laboratory, Prince Consort Road, London SW7 2AZ, UK \\ e-mail: k.nandra@imperial.ac.uk
}

Received 21 February 2003 / Accepted 15 September 2003

\begin{abstract}
We present a re-analysis of the simultaneous 30 day IUE/XTE observation of NGC 7469 done in 1996. Our main progress in this paper, in comparison to previous spectral analyses (Nandra et al. 1998, 2000), is to adopt and fit directly to the data a detailed model of the Comptonized spectrum. This firstly allows one to fit simultaneously the data from the UV to the hard X-ray band in a self-consistent way and secondly it gives direct constraints on the physical parameters of the disc-corona system, like the temperature and optical depth of the corona.

Our results are completely consistent with a slab geometry where all the observed UV emission is supposed to cross the corona but more photon-starved geometries also give acceptable fits. Whatever the geometry, the UV seed photon emission appears to be dominated by the reprocessing of the X-rays.

We also found very interesting correlations between the different model parameters, the most important one being the anticorrelation between the corona temperature $k T_{\mathrm{e}}$ and the UV flux $F_{\mathrm{UV}}$. Such an anticorrelation is clearly inconsistent with a fixed disc-corona configuration and suggests a variation of the geometry of the system. We also find a correlation between the corona optical depth and the X-ray flux which may reflect processes linked to the corona formation.

During the observations, NGC 7469 appears to accrete near its Eddington limit. This source could then be an example of magnetically dominated disc-corona system as recently proposed by Merloni (2003). Finally, these data strongly support the presence of a pair free corona.
\end{abstract}

Key words. galaxies: Seyfert - galaxies: individual: NGC 7469 - ultraviolet: galaxies - X-rays: galaxies

\section{Introduction}

The broad band opt-UV-X- $\gamma$-ray spectra of Seyfert galaxies is mainly dominated by two components: an optical/UV bump, which was interpreted early on early as the signature of cold material accreting onto a putative super massive black hole (Shields 1978; Malkan \& Sargent 1982), and a broader component, covering the soft-X to the hard-X/soft $\gamma$-ray energy band, with a rough power law shape generally cut-off above $100 \mathrm{keV}$.

These spectra are commonly interpreted in the framework of the reprocessing/upscattering models (e.g. Haardt \& Maraschi 1991, 1993). These models assume the presence of two phases, a cold (generally an accretion disc) and a hot (called the corona) one, radiatively linked one with each other: part of the cold emission, giving birth to the UV bump, is produced by the reprocessing of part of the hot emission. Inversely

Send offprint requests to: $\mathrm{P}$. O. Petrucci, e-mail: petrucci@obs.ujf-grenoble.fr the hot emission, at the origin of the broad X-ray component, is believed to be produced by Compton upscattering of the soft photons, emitted by the cold phase, on the coronal energetic electrons.

The presence of a high energy cut-off near $100 \mathrm{keV}$, first detected by SIGMA and OSSE in NGC 4151 (Jourdain et al. 1992; Maisack et al. 1993), appears to be common in Seyfert galaxies (Zdziarski et al. 1993; Gondek et al. 1996; Matt 2001; Perola et al. 2002). The presence of this component, and the lack of any annihilation lines (while poorly constrained) are generally believed to be the signature of the thermal nature of the coronal plasma (see however Malzac \& Jourdain 2000), the cut-off energy being then of the order of the coronal temperature. No cut-off but strong annihilation lines were indeed expected by the different non-thermal models develop in the 80-90s. However when taking better into account the feedback of the accelerated particles on the acceleration process (Done et al. 1990; Henri \& Pelletier 1991; Petrucci et al. 2001) 
non-thermal models also predict spectral shape in agreement with the observations.

Stronger and less ambiguous constraints on the nature of the coronal plasma are expected from variability studies. For example, in the case of a thermal hot corona in radiative equilibrium with the cold phase, the X-ray spectral shape is expected to harden (the photon index $\Gamma$ decreases) when the corona temperature (and thus the high energy cut-off) increases (Haardt et al. 1997). Inversely, in the case of a non-thermal plasma, a softening of the spectrum is expected when the cutoff energy increases to ensure the balance between the accelerated particles and the shocked gas pressures (Petrucci et al. 2001).

Such variations of the X-ray spectral shape may be produced by intrinsic changes of the corona properties (for example changes of the heating process efficiency) and/or by variation of the external environment like changes of the soft photons flux (and consequently of the corona cooling) produced by the cold phase. Simultaneous information in the UV and X-ray band are then needed to constrain the nature of the different processes acting in both phases.

Several observations have already suggested a link between the UV and/or the extreme UV and the X-ray emission in Seyfert galaxies (Clavel et al. 1992; Edelson et al. 1996; Marshall et al. 1997; Chiang et al. 2000; Uttley et al. 2000; Shemmer et al. 2001), showing strong correlations (with small or zero time lag) between the different fluxes, in good agreement with the reprocessing/upscattering interpretation. In some cases however, the X-rays and the optical/UV bands appear to be completely disconnected. For instance, the $\sim 1$ month simultaneous IUE/RXTE monitoring campaign on NGC 7469 performed in 1996, showed only poor correlations between the 2-10 keV and UV fluxes (Nandra et al. 1998, hereafter N98). Strong X-ray variations on small time scale were also detected but none in UV. Similar behavior has been observed in NGC 3516 (Edelson et al. 2000; Maoz et al. 2000, 2002). These last results ruled out apparently, and at least for these sources, the basic interpretation presented above.

The spectral analysis of the 1996 RXTE data of NGC 7469 by Nandra et al. (2000 hereafter N00) however permit to resolve these contradictions for this source. These authors indeed found that the X-ray spectral index in NGC 7469 was strongly correlated with the UV flux at zero time lag during the campaign. They interpret this result as a strong support for the X-rays being produced by Compton upscattering of the UV photons. In this framework, the observed variability of the UV flux is expected to directly modify the cooling rate of the hot corona, thus producing the observed X-ray spectral changes. The delays found between the RXTE hard X-rays with respect to the soft ones by Papadakis et al. (2001) also offers additional support to the hypothesis that the X-ray are most likely due to Comptonization of soft photons by hot, thermal electrons. The apparent disconnect between the X-ray and UV fluxes previously reported by N98, was then explained by the narrow band used in the X-ray (2-10 keV), which, in the presence of spectral variability, may result in incoherent behavior with respect to the UV one.
The spectral treatment done by N00 assumed a simple power law shape for the X-ray continuum. It is known however that the real Comptonization spectral shape may be significantly different from a power law (Haardt 1993; Svensson 1996; Petrucci et al. 2000). Moreover, the interpretation of the spectral variability in term of physical quantities, like the temperature and optical depth of the corona, is not straightforward with a phenomenological power law approximation and requires the use of approximate formulae. The aim of this paper is then to perform a re-analysis of the simultaneous IUE/XTE data by explicitly fitting the UV and X-ray continuum using a realistic Comptonization model. As just said, the first interest of this re-analysis is that we will have direct information on physical quantities characterizing the hot and cold phases. The broad band (from UV to hard X-rays) energy range of the Comptonization spectra also permit to fit simultaneously the IUE and RXTE data and to predict the spectral behavior at high energy (i.e. above $20 \mathrm{keV}$, the upper limit of our RXTE data) in a completely consistent way.

The paper is organized as follows. We first present the data and the model used in Sect. 2. We then show, in Sect. 3, the results we obtain fitting simultaneously the IUE and XTE data and we also present the most interesting correlations we found between the different model parameters. We discuss these different results in Sect. 4 and then conclude on this work in the last section.

\section{Observation and data analysis}

\subsection{The data}

Many of the details of the RXTE observations are reported in N98 and N00 and the reader can refer to these papers for more details. The "L7+activation" model was employed for the background. The standard selection criteria in the "rex" RXTE processing script has also been applied.

The background subtraction was however unreliable at high energies for sources as weak as NGC 7469 and the spectral analysis was therefore restricted to the $2-20 \mathrm{keV}$ energy band. Different response matrices were calculated for different times of the observation using PCARSP v2.37 but no significant differences were found between the derived spectra. We therefore used a single matrix for the entire observation.

The IUE data used here are described in Wanders et al. (1997). NGC 7469 has also been observed by the HST FOS instrument in the midst of the IUE monitoring (Kriss et al. 2000). The better quality of the HST data has allowed to disentangle the UV continuum more accurately from the broad wings of the emission lines and thus to identify four clean continuum windows free of any emission and/or absorption features, and centered on $1315,1485,1740$ and $1825 \AA$. We fit the data using XSPECv11.1. Since the XSPEC fitting tools need spectral bin larger than $1 \mathrm{eV}$, we have rescaled the UV fluxes in order to have 4 windows of $1 \mathrm{eV}$ band width. In the following, and to be comparable with the results of Wanders et al. (1997) and Kriss et al. (2000), the UV fluxes are given in $\operatorname{erg~s}^{-1} \mathrm{~cm}^{-2} \AA^{-1}$ and are measured in a bandwidth of $21 \AA$. 
Like in N00, the observation have been divided into $30 \mathrm{seg}$ ments of approximately 1 day duration with sufficient photon statistics to permit precise spectral analysis.

\subsection{The model}

We fit the data using, for the continuum, the thermal Comptonization model in slab geometry developed by F. Haardt (Haardt 1993; Haardt 1994; Haardt et al. 1997). Hereafter we will refer to this Anisotropic Comptonization Code as AC2. The code treats Comptonization in steady-state, i.e., energy balance is implicitly assumed, so that an unspecified heating term balances radiative losses. Given the temperature and optical depth of the corona, the angle-dependent spectra of a disc-corona system in plane parallel geometry is derived using an iterative scattering method, where the intrinsic anisotropy of the scattering is taken into account only in the first scattering order. The Compton recoil is also treated accurately. It includes also a reflection component described following White et al. (1988) and Lightman \& White (1988) and assuming neutral matter. The spectral shape of the reflected photons is averaged over angles. It is multiplied by a first normalization factor which depends on the inclination angle (see Ghisellini et al. 1994 for details). In addition, the usual $R$ normalization is left free to vary in the fit procedure, so that, for the given inclination angle, $R=1$ corresponds to a solid angle, subtended by the reflector, of $2 \pi$. The resulting Comptonization spectral shape generally is quite different from the simple cut-off power law approximation generally used to mimic Comptonization spectra. Moreover, due to anisotropy effects, the first scattering order is reduced in comparison to the other orders, and the observed spectral shape is better approximated by a broken power law, the energy of the break depending mainly on $k T_{\mathrm{e}}$ and $k T_{\mathrm{bb}}$. It is expected to be in the $2-10 \mathrm{keV}$ range for the parameter values we used (cf. Petrucci et al. 2000 for more details).

The fit parameters in $\mathrm{AC} 2$ are the temperature of the corona $k T_{\mathrm{e}}$, its optical depth $\tau$, the temperature of the disc $k T_{\mathrm{bb}}$ (assumed as a single temperature black body) and the reflection normalization $R$. There are then two extra parameters compared to the simple power law + reflection model used by N00. Other natural outputs of $\mathrm{AC} 2$ are the total (integrated over the solid angles) X-ray fluxes emitted by the corona (upward toward the observer and backward toward the disc) as well as the total UV flux emitted by the disc and the total flux Compton reflected at the disc surface. Different grids of this model are now available for public use as XSPEC table models ${ }^{1}$.

It is important to point out that no links between the Comptonized spectrum and the soft UV disc emission are imposed a priori. The model simply adjusts its parameters to fit the data. It is only a posteriori that the resulting best fit values can be interpreted in a physically motivated scenario. More specifically, the code gives the ratio between an arbitrarily normalized input cooling flux, $F_{\text {cool }}$, and the total emitted Compton spectrum, $F_{\mathrm{X}}$. That ratio can then be related to a specific geometry of the system, e.g. a homogeneous plane

\footnotetext{
${ }^{1}$ From http://pitto.mib.infn.it/haardt/ATABLES
}

parallel disc-corona system in radiative equilibrium as in Haardt \& Maraschi (1991), in which case it must be $F_{\text {cool }} \simeq F_{\mathrm{X}}$. This balance is imposed by the feed-back of the corona on the disc and vice versa in the absence of a dissipation mechanism intrinsic to the disc and is independent on the heating rate, provided it heats the electrons in the corona. A doubling of the heating rate doubles $F_{\mathrm{X}}$ and $F_{\text {cool }}$ but does not change their ratio. If $F_{\mathrm{X}} \gtrsim F_{\text {cool }}$, a photon starved configuration is required for instance a spherical corona. In the most general case, $F_{\text {cool }}$ is a fraction of the observed UV flux $F_{\mathrm{UV}}$. In fact, the condition $F_{\mathrm{UV}}=F_{\text {cool }}$ is strictly valid only if all UV photons cross (and hence cool) the corona. In general $F_{\mathrm{UV}} \geq F_{\text {cool }}$. Thus, once the best fit parameters are obtained, one should seek for a selfconsistent (in terms of physics and geometry) configuration of the disc+corona system.

$\mathrm{AC} 2$ also does not treat the ionization and thermal balance of the cold phase at all. This has certainly some impact on the broad band spectral shape expected from such a model as shown by Malzac et al. (2003), leading, for instance, to higher corona temperature and harder spectra in comparison to the "blackbody + neutral reflection" approximation used here. We believe however that these effects have no strong influence on the spectral variability behaviors studied in this paper.

We have also the possibility of using a semi-spherical geometry for the corona. We have checked however that the results presented below do not significantly change in comparison to the slab configuration (the main change being the larger value of the optical depth for a given slope of the X-ray power law). We thus restrict our analysis to the slab geometry.

We used the UVRED model for the reddening, fixing the extinction to the best fit value of Kriss et al. (2000) i.e. $E(B-V)=$ 0.12 . We fixed the column density to the galactic one i.e. $N_{\mathrm{H}}=4.8 \times 10^{20} \mathrm{~cm}^{-2}$. For the neutral iron line, we simply used a Gaussian (ZGAUSS model of XSPEC), fixing the Gaussian energy and width to $6.4 \mathrm{keV}$ and $0.01 \mathrm{keV}$ respectively. The Gaussian normalization was let free to vary. Finally we assume a relative normalization of 1 between the IUE and XTE data (we have checked that normalizations of 0.9 or 1.1 do not significantly change the results).

\section{Simultaneous IUE and XTE fits}

\subsection{Fit results}

We have reported in Table 1 the best fit values of the different model parameters, i.e. $k T_{\mathrm{e}}, \tau, k T_{\mathrm{bb}}$ and $R$, for 29 daily segments fitting simultaneously the IUE and XTE data. We do not include the first segment obtained at the beginning of the campaign, JD-24 400245.104 , since no IUE data were available. We have also reported in the table the UV (at $1315 \AA$ ) and $\mathrm{X}$-ray (in the $2-10 \mathrm{keV}$ range) fluxes, and the $\chi^{2}$ values. We have plotted in Fig. 1 the data and the corresponding best fit models for two different states of NGC 7469 (a soft one corresponding to segment 2 and a hard one corresponding to segment 9).

We obtained acceptable fits for each segment and the total $\chi^{2}=1528$ for 1334 d.o.f. The use of the IUE and XTE data provide relatively good constraints on the different parameters 
Table 1. Best fit values of our model parameters $k T_{\mathrm{e}}, \tau, k T_{\mathrm{bb}}$ and $R$ obtained when fitting the XTE and IUE data simultaneously. We have also reported the X-ray and UV (at $1315 \AA$ ) fluxes (in $\mathrm{erg} \mathrm{s}^{-1} \mathrm{~cm}^{-2}$ and $\mathrm{erg} \mathrm{s}^{-1} \mathrm{~cm}^{-2} \AA^{-1}$ respectively).

\begin{tabular}{|c|c|c|c|c|c|c|c|}
\hline $\begin{array}{c}\text { Day } \\
(\mathrm{TDJ}+24400000)\end{array}$ & $\begin{array}{c}k T_{\mathrm{e}} \\
(\mathrm{keV})\end{array}$ & $\tau$ & $\begin{array}{l}k T_{\mathrm{bb}} \\
(\mathrm{eV})\end{array}$ & $R$ & $\begin{array}{c}F_{2-10} \\
\left(\times 10^{-11}\right)\end{array}$ & $\begin{array}{c}F_{1315} \\
\left(\times 10^{-14}\right)\end{array}$ & $\begin{array}{c}\chi^{2} \\
\text { (46 d.o.f.) }\end{array}$ \\
\hline 246.177 & $286_{-10}^{+20}$ & $0.067_{-0.009}^{+0.007}$ & $2.0_{-0.1}^{+0.2}$ & $1.0_{-0.1}^{+0.1}$ & 3.44 & 4.90 & 50.0 \\
\hline 247.247 & $317_{-26}^{+20}$ & $0.051_{-0.004}^{+0.010}$ & $2.1_{-0.1}^{+0.1}$ & $1.0_{-0.2}^{+0.2}$ & 2.87 & 5.09 & 49.3 \\
\hline 248.316 & $327_{-26}^{+10}$ & $0.058_{-0.004}^{+0.010}$ & $2.1_{-0.1}^{+0.1}$ & $0.9_{-0.1}^{+0.2}$ & 3.69 & 4.76 & 62.4 \\
\hline 249.386 & $317_{-20}^{+15}$ & $0.062_{-0.006}^{+0.009}$ & $2.0_{-0.1}^{+0.2}$ & $0.8_{-0.2}^{+0.2}$ & 3.79 & 4.63 & 67.6 \\
\hline 250.456 & $342_{-20}^{+15}$ & $0.058_{-0.004}^{+0.007}$ & $1.9_{-0.1}^{+0.1}$ & $0.6_{-0.1}^{+0.1}$ & 3.89 & 4.15 & 34.5 \\
\hline 251.579 & $363_{-20}^{+20}$ & $0.051_{-0.004}^{+0.005}$ & $1.9_{-0.2}^{+0.2}$ & $1.1_{-0.2}^{+0.2}$ & 3.38 & 3.74 & 65.3 \\
\hline 252.631 & $347_{-26}^{+20}$ & $0.054_{-0.005}^{+0.007}$ & $1.9_{-0.2}^{+0.1}$ & $1.3_{-0.2}^{+0.1}$ & 3.16 & 3.63 & 41.3 \\
\hline 253.676 & $419_{-31}^{+36}$ & $0.038_{-0.003}^{+0.004}$ & $1.9_{-0.2}^{+0.1}$ & $1.3_{-0.5}^{+0.3}$ & 2.53 & 3.17 & 50.4 \\
\hline 254.714 & $342_{-20}^{+20}$ & $0.057_{-0.006}^{+0.007}$ & $1.9_{-0.2}^{+0.2}$ & $0.9_{-0.2}^{+0.2}$ & 3.15 & 3.40 & 45.0 \\
\hline 255.777 & $322_{-61}^{+26}$ & $0.055_{-0.007}^{+0.010}$ & $2.0_{-0.1}^{+0.2}$ & $1.5_{-0.3}^{+0.2}$ & 2.80 & 3.90 & 62.0 \\
\hline 256.811 & $337_{-31}^{+20}$ & $0.050_{-0.005}^{+0.008}$ & $2.0_{-0.1}^{+0.1}$ & $1.9_{-0.2}^{+0.2}$ & 2.75 & 3.78 & 45.2 \\
\hline 257.840 & $342_{-41}^{+15}$ & $0.048_{-0.004}^{+0.012}$ & $2.0_{-0.1}^{+0.2}$ & $1.8_{-0.4}^{+0.6}$ & 2.74 & 4.00 & 43.9 \\
\hline 258.865 & $322_{-26}^{+20}$ & $0.050_{-0.006}^{+0.009}$ & $2.1_{-0.1}^{+0.2}$ & $1.8_{-0.3}^{+0.2}$ & 2.53 & 4.40 & 49.9 \\
\hline 259.914 & $281_{-20}^{+26}$ & $0.063_{-0.009}^{+0.014}$ & $2.1_{-0.2}^{+0.1}$ & $2.0_{-0.2}^{+0.3}$ & 2.86 & 4.64 & 43.4 \\
\hline 260.914 & $322_{-36}^{+26}$ & $0.049_{-0.005}^{+0.013}$ & $2.1_{-0.1}^{+0.1}$ & $1.7_{-0.2}^{+0.4}$ & 2.60 & 4.65 & 56.6 \\
\hline 261.918 & $312_{-26}^{+50}$ & $0.053_{-0.005}^{+0.005}$ & $2.1_{-0.1}^{+0.1}$ & $1.5_{-0.2}^{+0.2}$ & 2.82 & 4.85 & 42.8 \\
\hline 262.882 & $286_{-15}^{+31}$ & $0.064_{-0.009}^{+0.011}$ & $2.1_{-0.1}^{+0.1}$ & $1.3_{-0.2}^{+0.2}$ & 3.36 & 5.14 & 56.3 \\
\hline 263.883 & $286_{-26}^{+10}$ & $0.065_{-0.005}^{+0.011}$ & $2.0_{-0.1}^{+0.2}$ & $1.4_{-0.2}^{+0.1}$ & 3.28 & 5.04 & 55.8 \\
\hline 264.917 & $307_{-15}^{+15}$ & $0.060_{-0.005}^{+0.011}$ & $2.0_{-0.1}^{+0.2}$ & $0.8_{-0.2}^{+0.1}$ & 3.51 & 5.09 & 40.6 \\
\hline 265.953 & $296_{-10}^{+20}$ & $0.067_{-0.007}^{+0.008}$ & $2.1_{-0.2}^{+0.2}$ & $1.2_{-0.1}^{+0.1}$ & 3.90 & 4.95 & 64.9 \\
\hline 267.019 & $327_{-15}^{+15}$ & $0.062_{-0.005}^{+0.007}$ & $2.1_{-0.2}^{+0.1}$ & $0.8_{-0.1}^{+0.2}$ & 4.02 & 4.50 & 61.8 \\
\hline 268.084 & $301_{-20}^{+10}$ & $0.072_{-0.009}^{+0.006}$ & $1.9_{-0.4}^{+0.4}$ & $1.1_{-0.1}^{+0.2}$ & 3.92 & 3.99 & 53.8 \\
\hline 269.155 & $358_{-26}^{+36}$ & $0.058_{-0.004}^{+0.003}$ & $1.8_{-0.3}^{+0.3}$ & $0.5_{-0.2}^{+0.2}$ & 3.83 & 3.53 & 58.4 \\
\hline 270.222 & $347_{-15}^{+26}$ & $0.056_{-0.007}^{+0.006}$ & $2.0_{-0.2}^{+0.1}$ & $1.1_{-0.3}^{+0.2}$ & 3.31 & 3.52 & 43.8 \\
\hline 271.282 & $383_{-26}^{+15}$ & $0.050_{-0.003}^{+0.006}$ & $1.8_{-0.2}^{+0.2}$ & $0.7_{-0.2}^{+0.2}$ & 3.38 & 3.32 & 58.0 \\
\hline 272.361 & $373_{-20}^{+31}$ & $0.047_{-0.004}^{+0.006}$ & $2.0_{-0.2}^{+0.1}$ & $1.5_{-0.2}^{+0.3}$ & 2.71 & 3.24 & 59.8 \\
\hline 273.417 & $322_{-10}^{+31}$ & $0.057_{-0.008}^{+0.007}$ & $1.9_{-0.2}^{+0.1}$ & $1.9_{-0.2}^{+0.2}$ & 2.66 & 3.25 & 62.5 \\
\hline 274.488 & $312_{-26}^{+15}$ & $0.065_{-0.005}^{+0.013}$ & $2.2_{-0.2}^{+0.1}$ & $1.3_{-0.1}^{+0.2}$ & 3.21 & 3.71 & 41.4 \\
\hline 275.547 & $266_{-46}^{+15}$ & $0.079_{-0.011}^{+0.009}$ & $2.0_{-0.1}^{+0.2}$ & $1.4_{-0.1}^{+0.1}$ & 3.22 & 4.21 & 61.7 \\
\hline
\end{tabular}

of the model. Indeed, the UV data allow to constrain well $k T_{\mathrm{bb}}$. Moreover, in Comptonization models, and for a given geometry, the ratio between the UV bump maximum and the X-ray "plateau" (i.e. the X-ray flux near $0.1 \mathrm{keV}$ ) is mainly controlled by the corona optical depth. Combined with the X-ray slope in the $2-10 \mathrm{keV}$ range it then permits relatively good determination of $\tau$ and $k T_{\mathrm{e}}$ and thus of the X-ray continuum up to the hard $\mathrm{X}$-ray/soft $\gamma$-ray band. Consequently, the reflection component is also well constrained even if the XTE data permit precise spectral study only below $20 \mathrm{keV}$.

\subsection{Light curves of fit parameters}

We have plotted the light curves of the different model parameters on the bottom part of Fig. 2, as well as the total (above $0.1 \mathrm{keV}$ ) X-ray flux predicted by the AC2 code. We have also plotted the $2-10 \mathrm{keV} X$-ray and UV (at $1315 \AA$ ) light curves and the photon index one, obtained by N00, on the top of the figure.

We have tested the significance of the variability of each parameter. Fitting the different light curves with a constant gives a $\chi^{2}$, for 29 d.o.f., of 57.6, 54.9, 8.3 and 128.5 for $k T_{\mathrm{e}}, \tau, k T_{\mathrm{bb}}$ and $R$, respectively. Except $k T_{\mathrm{bb}}$, the other parameters are thus clearly variable (significant at $>99 \%$ confidence).

A more sensitive way to detect parameters variations is the use of the F-test. For that purpose, we fit again the data but fixing each of the parameters $\left(k T_{\mathrm{e}}, \tau, k T_{\mathrm{bb}}\right.$ and $\left.R\right)$ to its weighted average value (i.e. $322 \mathrm{keV}, 0.054,2 \mathrm{eV}$ and 1.1 respectively). By comparing the total $\chi^{2}$ of these fits to the $\chi^{2}$ when all parameters were free, we derived $F$-values of $4.3,3.9,1.2$ and 4.9 for 29 additional parameters. Again, it implies highly significant changes in $k T_{\mathrm{e}}, \tau$ and $R(>99 \%)$ but no significant changes for $k T_{\mathrm{bb}}$. The black body temperature $k T_{\mathrm{bb}}$ is thus consistent 


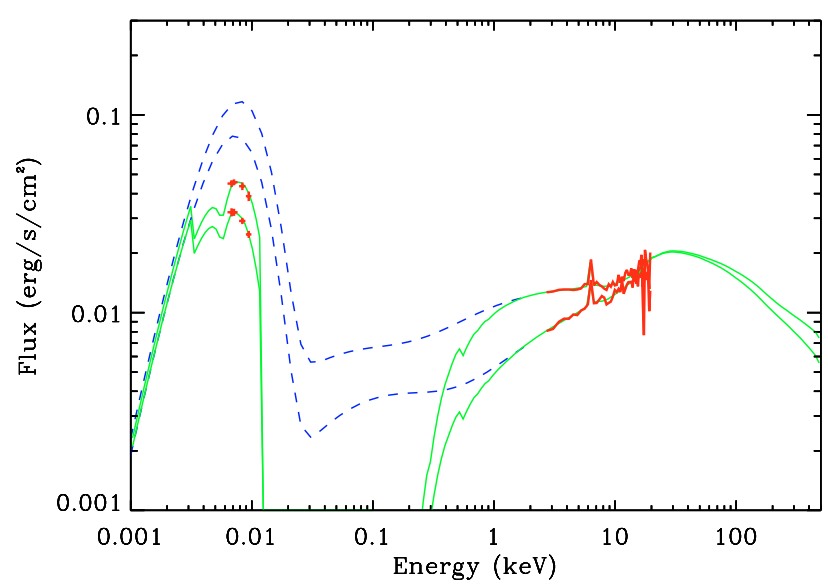

Fig. 1. Data and best fit model corresponding to a soft (segment 2) and hard (segment 9) state of NGC 7469. The data are in thick (red) lines, the unfolded model (including the reddening and the neutral hydrogen absorption) in thin solid (green) lines, and the unfolded model (without reddening or neutral hydrogen absorption) in dashed (blue) lines.

Table 2. The Spearman (rank) correlation coefficients are reported in the upper right portion of the table and the Pearson linear ones in the lower left part. The correlations have 29 points. The correlation coefficients with formal chance probabilities less than $1 \%$ (i.e. with correlation coefficient $>0.46$ ) are written in bold faces.

\begin{tabular}{ccccccc}
\hline \hline & $k T_{\mathrm{e}}$ & $\tau$ & $R$ & $F(2-10)$ & $F_{1315}$ & $F_{\text {line }}$ \\
\hline$k T_{\mathrm{e}}$ & $\ldots$ & $\mathbf{- 0 . 6 5}$ & -0.26 & -0.15 & $\mathbf{- 0 . 7 3}$ & -0.03 \\
$\tau$ & $\mathbf{- 0 . 7 9}$ & $\ldots$ & -0.30 & $\mathbf{0 . 6 7}$ & 0.37 & 0.23 \\
$R$ & -0.22 & -0.21 & $\ldots$ & $\mathbf{- 0 . 7 9}$ & -0.05 & -0.31 \\
$F(2-10)$ & -0.20 & $\mathbf{0 . 5 9}$ & $\mathbf{- 0 . 7 9}$ & $\ldots$ & 0.35 & 0.40 \\
$F_{1315}$ & $\mathbf{- 0 . 6 7}$ & 0.35 & -0.07 & 0.30 & $\ldots$ & -0.05 \\
$F_{\text {line }}$ & -0.16 & 0.33 & -0.34 & 0.43 & 0.04 & $\ldots$ \\
\hline
\end{tabular}

with a constant and we choose to fix it, in the following, to the mean value of $2 \mathrm{eV}$.

We finally remark that the total X-ray flux (above $0.1 \mathrm{keV}$ ) predicted by our model also varies similarly to the observed $2-10 \mathrm{keV}$ flux.

\subsection{Correlations}

We have reported in Table 2 the results of the linear (Pearson) and rank (Spearman) correlation tests between the different model parameters, assuming no time lags.

The X-ray flux is apparently correlated, with significances $>99 \%$, with the coronal optical depth $\tau$ and anticorrelated with $R$. This anticorrelation was not observed by N00. We also found a correlation between the X-ray and line fluxes but not as strong as N00. Concerning the UV flux, it appears strongly anti-correlated with the coronal temperature $k T_{\mathrm{e}}$. Finally, we found a strong anti-correlation between $k T_{\mathrm{e}}$ and $\tau$. We have plotted the most significant correlations in Figs. 3 and 4 i.e $\tau$ and $R$ versus $F_{2-10}, k T_{\mathrm{e}}$ versus $F_{1315}$, and $k T_{\mathrm{e}}$ versus $\tau$.

As already remarked by $\mathrm{N} 00$, the cross correlation of light curves that have a "red noise" character (i.e. where the
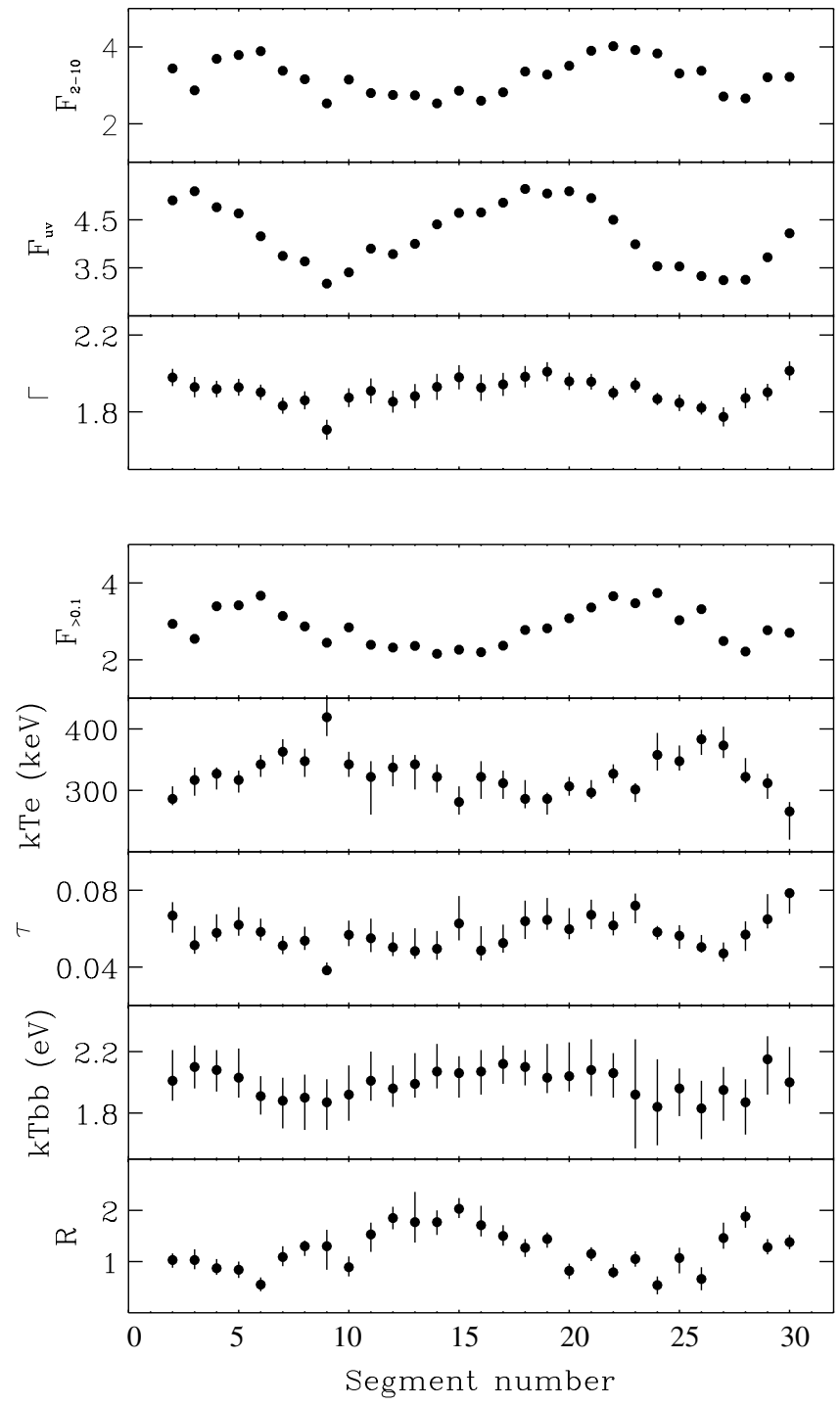

Fig. 2. The light curves of the different parameters of the model (i.e. $k T_{\mathrm{e}}, \tau, k T_{\mathrm{bb}}$ and $R$ ) are plotted in the bottom part of the figure as well as the total (above $0.1 \mathrm{keV}$ ) X-ray flux predicted by the AC2 code (in $10^{-10} \mathrm{erg} \mathrm{s}^{-1} \mathrm{~cm}^{-2}$ ). For comparison, we have also plotted, at the top, the observed UV flux at $1315 \AA$ (in $10^{-14} \mathrm{erg} \mathrm{s}^{-1} \mathrm{~cm}^{-2} \AA^{-1}$ ), the 2-10 $\mathrm{keV} X$-ray flux (in $10^{-11} \mathrm{erg} \mathrm{s}^{-1} \mathrm{~cm}^{-2}$ ) and the photon index light curve obtained by NOO.

variability power scales as $f^{-\alpha}$ ), can produce artificially high correlation coefficients values (Welsh 1999; Maoz et al. 2000). We have thus tested the significance of our results by simulating a number of X-ray and UV light curves with "red noise" power spectra. We adopt a value of $\alpha$ of 1.3 and 1.9 for the power law index of the X-ray and UV power-density spectra respectively (cf. N98 and N00).

In 200 trials of 29 points, we never obtained Spearman or Pearson correlation coefficients as high as that observed in the real data. We thus conclude that the correlations shown in bold in Table 2 are unlikely to arise by chance. We discuss these different correlations in the following. 

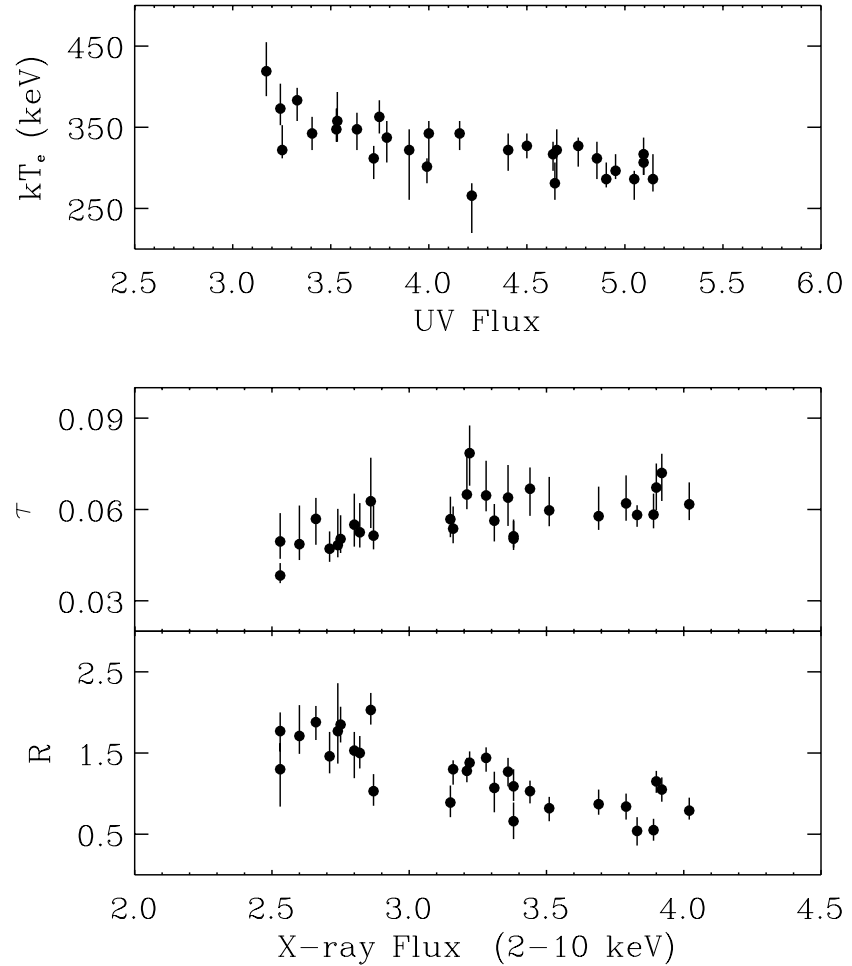

Fig. 3. Top: coronal temperature vs. UV flux at $1315 \AA$ (in erg s $\mathrm{s}^{-1} \mathrm{~cm}^{-2} \AA^{-1}$ ). Bottom: coronal optical depth $\tau$ and reflection normalization $R$ vs. $2-10 \mathrm{keV}$ flux (in erg s $\mathrm{s}^{-1} \mathrm{~cm}^{-2}$ ).

\subsection{1. $k T_{\mathrm{e}}$ versus $F_{\mathrm{UV}}$}

The coronal temperature appears strongly anticorrelated with the UV flux ( $F_{1315}$ mimics very well the total UV flux variations predicted by our model and in the following we will now only use the term $F_{\mathrm{UV}}$ instead of $F_{1315}$ ). We found a rank Spearman coefficient of 0.73 while the highest coefficient obtained with our simulations, using 200 trials, was $\sim 0.5$ ( 0.6 for 400 trials). This anti-correlation clearly corresponds to the $\Gamma$ vs. $F_{\mathrm{Uv}}$ correlation found by N00.

These authors interpret this correlation as a strong support for Comptonization models where any "primary" increase of the UV seed photons (not accompanied by an increase of the coronal heating rate) is generally expected to produce an increase of the cooling of the corona and thus a steepening of the spectrum and a decreasing of the corona temperature. We will see in the following that, in the context of coupled disc-corona models where the UV arises from reprocessing, a geometrical and/or energetic change of the disc-corona configuration is required.

\subsection{2. $\tau$ versus $F_{2-10}$}

We find a strong correlation between the coronal optical depth and the X-ray emission. As already noted in Sect. 2.2, in the code we used there is no link imposed a priori between $\tau$ and the total X-ray flux $F_{X}$ and consequently between $\tau$ and $F_{2-10}$. Such correlation is not simply explained in the Comptonization model framework and may reflect a process of different nature.

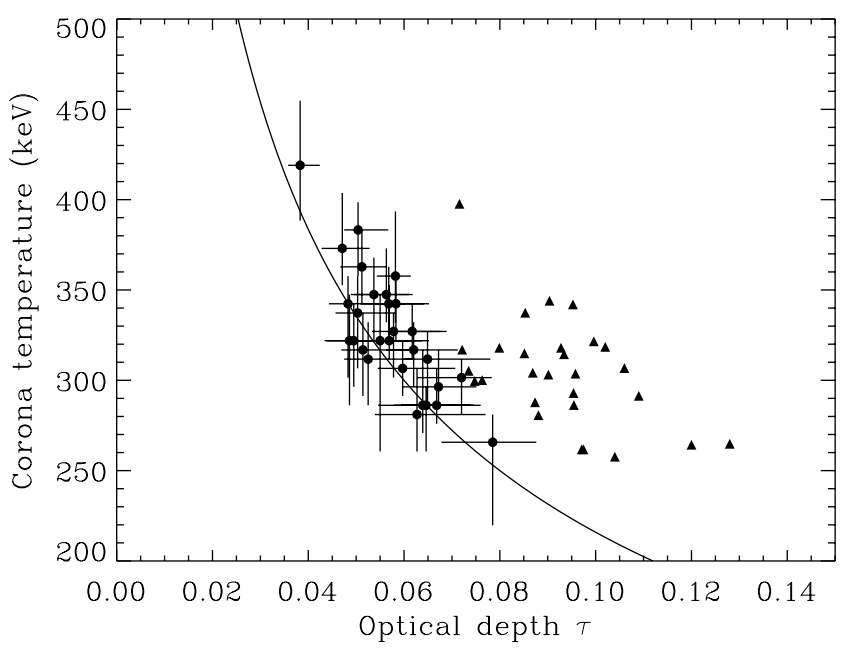

Fig. 4. Coronal temperature vs. coronal optical depth. Filled circles (with errors) have been obtained assuming that all the observed UV flux has crossed the corona. Filled triangles correspond to the case where only half of the observed UV flux is supposed to have crossed the corona. The errors have been omitted in this case for clarity. The relation expected in the case of a slab corona above a passive disc has been overplotted in solid line (cf. Sect. 3.3.3 for details).

For instance, we expect that part of the X-ray flux produced by the corona illuminates the surrounding cold thick material, seed of soft photons. It is also possible that the hot corona is produced by e.g. evaporation of part of this cold matter or by magnetic buoyancy inside the cold matter (Meyer \& Meyer-Hofmeister 1994; Meyer et al. 2000; Liu et al. 2002; Merloni \& Fabian 2002; Merloni 2003). Then variations of the $\mathrm{X}$-ray flux may likely modify the properties of the upper layers of the accretion disc, and thus influence the corona formation. For instance, thermal instabilities are known to exist in illuminated plasma (Różańska \& Czerny 1996; Nayakshin et al. 2000), and the degree of ionization and temperature of the disc surface may rapidly increase in response to an increase of the illuminating flux. It may then favor the evaporation of part of the disc matter, increasing consequently the corona optical depth (but see Sect. 4.3).

\subsection{3. $k T_{\mathrm{e}}$ vs. $\tau$}

We have reported in Fig. 4 the coronal optical depth $\tau$ versus the coronal temperature $k T_{\mathrm{e}}$ deduced from our fits. A strong anti-correlation is clearly visible. Such anti-correlation is indeed expected in the case of a corona-disc configuration in radiative equilibrium. It depends however on the real geometry of the system (Haardt \& Maraschi, 1993; Stern et al. 1995; Malzac et al. 2001). The relation expected in the case of a slab corona above a passive disc has been overplotted in solid line in Fig. 4. The data seem to be in good agreement with the theoretical predictions, even if they slightly stand above the solid line and present some dispersion.

It is worth noting that in our fitting procedure, we have assumed that all the observed UV emission, whatever its origin, has crossed and cooled the corona. It is however possible that part of the disc emission does not interact with the corona, like 
in the case of a patchy or spherical corona. We have simulated such a configuration by adding a black body component to the model used to fit the data. Its temperature was fixed to $2 \mathrm{eV}$ and its normalization was fixed so that the corresponding flux (at $1315 \AA$ ) was half the observed mean UV flux. In this way, we impose that a fixed part of the UV emission does not cross and cool the corona. The corresponding values of $\tau$ and $k T_{\mathrm{e}}$ have been overplotted with triangles in Fig. 4. We omit the error bars for clarity. The triangles are clearly above the solid line, as expected for a more photon starved configuration. The total $\chi^{2}$ that we obtained with this model is 1592 still for 1334 d.o.f., slightly larger than without the addition of a black body component, but statistically equivalent. A geometry more photonstarved than the simple slab corona is thus also in agreement with the observations.

It is worth noting that the addition of a constant black body component does not substantially modify the results presented above. In particular, we still found correlation coefficients of the same order of magnitude than those reported in Table 2.

\subsection{4. $F_{\mathrm{K}_{\alpha}}, R$ versus $F_{2-10}$}

We found only a weak correlation between the line flux and the X-ray flux (cf. NOO who found a stronger correlation) but we did find an anti-correlation, not noted by N00, between $R$ and $F_{2-10}$.

It is known that the measurement of the reflection component depends on the assumed model for the continuum. For example, large differences are expected between a simple power law shape and a real Comptonization model (Petrucci et al. 2001). It may then explain the difference between our results and those of N00. It does not explain however why $R$ is anticorrelated with the $2-10 \mathrm{keV}$ flux. Moreover these results are relatively difficult to explain in the standard view since both the line and the reflection component are expected to arise from the same region and thus to vary in the same way.

It is possible that part of the reflection components is emitted close to the central region while the other part may be produced by a remote reflector (indeed the presence of a distant reflector may explain the $R-F_{2-10}$ anticorrelation). In this case, and in the presence of flux and spectral variability, like for NGC 7469, the measurement of $R$ and $F_{\text {line }}$ may not be very reliable and meaningful (Malzac \& Petrucci 2002) especially with the limited band pass and spectral resolution of XTE and the increasing noise at high energy (near $20 \mathrm{keV}$ ). In agreement with N00, we thus believe that the derived values of $R$ and $F_{\text {line }}$ may not be truly representative of the strength and spectral shape of the real reflection components in NGC 7469. We will thus not discuss this parameter in the following.

\section{Discussion}

Important results have emerged from this analysis. Firstly, we obtained very acceptable fits, from the UV to the X-ray range, of the 29 daily spectra of NGC 7469 using a realistic thermal Comptonization model. This model is able to explain the spectral shape of the different UV/X-ray daily spectra but also the UV and X-ray flux variability. In agreement with the conclusion of N00 (see also Chiang 2002), the absence of a clear correlation between the UV and X-ray fluxes, as reported by $\mathrm{N} 98$, was thus clearly misleading. It simply results from the complex flux and spectral variability of this source which may produce, when observed in narrow energy bands, relatively unexpected behaviors. Berkley et al. (2000) have failed to reproduce the UV and X-ray light curves of NGC 7469 using a simple model where an X-ray point source illuminates an infinite accretion disc. This model does not consider spectral changes at all and thus cannot reflect the intrinsic spectral complexity of comptonization models. We believe that this is the main reason of the results they obtained.

We also note at this point that our best fit results reported in Table 1 are quantitatively different from those obtained by Chiang (2002) fitting the same data. We also believe that the differences are certainly due to the approximate model used by this author to mimic thermal Comptonization spectra. For example, for corona optical depth as small as the ones he found (cf. his Fig. 5), a thermal Comptonization spectrum is formed of well separated bumps of different scattering orders and a simple cut-off power law is a really poor approximation.

Secondly, we found very interesting correlations between the UV/X-ray fluxes and the model parameters, the most important one being the anticorrelation between the UV flux and the corona temperature. It is the equivalent of the $F_{\mathrm{UV}}-\Gamma$ correlation found by $\mathrm{N} 00$, fitting the data with a simple cut-off power law model. This anticorrelation can be simply explained in the framework of thermal Comptonization models. Indeed, the increase of the UV soft photons flux, if "primary", generally means an increase of the coronal cooling. This then produces a decrease of the corona temperature and a softening of the spectrum as observed.

However, important results stem from this interpretation. First, the decrease of the corona temperature due to the increase of the coronal cooling is completely true in the case of a pairfree corona. Indeed, for a pair-dominated corona, the increase of the cooling would correspond to an increase of the corona temperature rather than a decrease (Ghisellini \& Haardt 1994). As discussed in Sect. 4.4, there are actually strong indications supporting the presence of a pair-free corona in NGC 7469, thus justifying the above interpretation.

More importantly, we note that the $F_{\mathrm{UV}}-k T_{\mathrm{e}}$ anticorrelation cannot be reconciled with a fixed disc-corona configuration in radiative balance. As explained in Sect. 2.2, such configuration corresponds to a fixed ratio $F_{\mathrm{X}} / F_{\mathrm{UV}}$. But there is a priori no link between $F_{\mathrm{UV}}$ and the corona parameters like its temperature or optical depth. $k T_{\mathrm{e}}$ and $\tau$ have only to adjust themselves in order to insure a constant heating/cooling ratio (cf. Sect. 2.2). There is thus no reason for $k T_{\mathrm{e}}$ and/or $\tau$ to change, whatever the value of $F_{\mathrm{UV}}$.

As discussed in the following sections, the $F_{\mathrm{UV}}-k T_{\mathrm{e}}$ anticorrelation necessarily suggests a change of the geometry of the disc-corona configuration. 


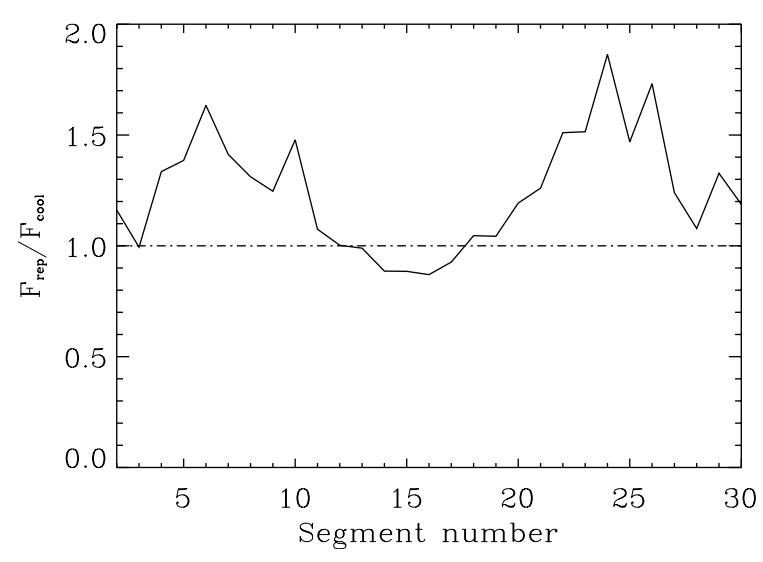

Fig. 5. Ratio of X-ray flux expected to be reprocessed by the disc, $F_{\text {rep }}$ and the disc flux which crosses and actually cools the corona $F_{\text {cool }}$ (solid line). The theoretical prediction in the case of a slab corona above a passive disc in radiative equilibrium is plotted in dashed line.

\subsection{UV emission dominated by reprocessing}

In the Comptonization code we used, there is no constraint, a priori, on the origin of the UV emission. It may be dominated by the intrinsic disc emission, by the reprocessed one, or both may be of the same order. A check can be made a posteriori by comparing the $\tau-k T_{\mathrm{e}}$ values with the theoretical expectations as we have done in Sect. 3.3.3 and Fig. 4. If the intrinsic disc emission were dominant, the corona should be overcooled in comparison to a corona above a passive disc, and the $\tau-k T_{\mathrm{e}}$ values should be in the lower right part of Fig. 4, below the solid line, which is not what we obtain.

Another way to see this is to compare the X-ray flux expected to be reprocessed by the disc, $F_{\text {rep }}$, and the UV flux which crosses and effectively cools the corona $F_{\text {cool }}$. The former is simply the difference between the flux emitted by the corona backward toward the disc and the flux Compton reflected by the disc. For the latter, and in the case of a slab corona completely covering the disc, $F_{\text {cool }}$ is equal to the total disc emission (this does not correspond to the observed UV emission, since part of the disc emission is comptonized in the corona). Both $F_{\text {rep }}$ and $F_{\text {cool }}$ are natural outputs of the Comptonization model used to fit the data. We have plotted in Fig. 5, the corresponding ratio $F_{\text {rep }} / F_{\text {cool }}$. This ratio appears to be generally larger than 1 . It is also variable between 1 and 1.8 and this point is discussed in the next section. If some part of the UV emission crossing the corona was intrinsic to the disc we would expect this ratio to be smaller than 1 which is not the case.

These results thus suggest that, in the case of NGC 7469, the intrinsic disc emission is negligible in comparison to the reprocessed one. This conclusion is in complete agreement with that of N00. It also agrees with the observed delays, increasing with wavelength, observed between different UV bands and interpreted as the different light travel time between the illuminating X-ray source and the disc reprocessing regions (Wanders et al. 1997; Kriss et al. 2000).

Still concerning Fig. 5, we note that, starting from the epoch (segm n. 15) where the ratio $F_{\text {rep }} / F_{\text {cool }}$ is nearly 1 , as expected for the slab configuration, this ratio reaches its maxima in near correspondence with the maxima of the X-ray light curve (near minima for the UV fluxes). One can thus interpret the "oscillation" as a change in geometry whereby the fraction of reprocessed photons which re-enter and cool the corona varies.

\subsection{Corona geometry variations as the origin of the observed variability}

Simulations of Malzac \& Jourdain (2001) show that the time scale for a disc-corona system to reach radiative equilibrium, after rapid radiative perturbation in one of the two phases, is of the order of a few corona light crossing times i.e. well smaller than a day. We can thus (reasonably) assume that each of the 29 daily average spectra and fluxes of the observation of NGC 7469 correspond to a disc-corona system where hot and cold phases are in radiative balance.

We have also shown in the previous section that the disc emission is apparently dominated by the $\mathrm{X}$-ray reprocessing. In this case, a fixed disc-corona configuration in radiative equilibrium should correspond to a constant $F_{\mathrm{X}} / F_{\mathrm{UV}}$ ratio (cf. Sect. 2.2) contrary to what we observe in Fig. 5. We are thus bring to the conclusion that the disc-corona geometry necessarily varies during the total observation, producing the observed spectral and flux variability.

It is not clear how the geometry really varies especially since we have no real constraint on the geometry itself. However, the fact that the X-ray emission is apparently the primary source of radiation (the UV being only produced by reprocessing), but also of variability (the total X-ray flux, above $0.1 \mathrm{keV}$, is indeed predicted to vary along the observation, cf. Fig. 2), naturally suggests that the change of the disc-corona geometry is actually a change of the corona geometry: the larger/smaller the corona, the stronger/weaker the $\mathrm{X}$-ray emission.

We then propose the following scenario. Suppose that the origin of the day time scale variability is indeed a change of the global corona geometry. Suppose for example that the corona, which covers initially a large part of the disc, then becomes more patchy. Then the more patchy the corona, the hotter the mean corona temperature becomes and the harder the X-ray spectrum is. At the same time, the corona covering factor decreasing, the reprocessed flux, and consequently the global UV flux, decreases. This scenario thus simply explains the observed $k T_{\mathrm{e}}-F_{\mathrm{UV}}$ anticorrelation.

Moreover we may naturally expect the optical depth of the corona to depend on the disc X-ray illumination (which mainly occurs below the corona blobs) as already suggested in Sect. 3.3.2. The variation of the X-ray flux impinging on the disc could likely modify the structure of the upper layers of the accretion disc and thus have an impact on the corona formation process. A strong X-ray emission would then help the evaporation of the accretion disc material thus increasing the corona optical depth. This could also explain the correlation between the corona optical depth and the X-ray flux.

We of course do not assert that this scenario is unique. We easily imagine that the change of the disc-corona geometry is certainly more complex, and may also imply changes of the 
disc itself. For instance, its inner region may become geometrically thick and optically thin, due to thermal instabilities (however see next section), or its inner radius may vary with time. Consequently, it would modify the UV flux entering the corona as well as the reflection component. The time scale for such processes to occur is however expected to be quite long in comparison to what we observe. Anyway, whatever the scenario proposed, the present analysis strongly requires a change of the disc-corona configuration. On the other hand, this appears to be a very plausible origin for the flux and spectral variability observed in NGC 7469.

\subsection{A magnetically dominated corona in NGC 7469?}

The mass of the black hole supposed to be present in the central region of NGC 7469 has been estimated in between $10^{6}$ and $10^{7}$ solar masses. Precise measurements, using different methods, favor a mass of about $7 \times 10^{6} M_{\odot}$ (Collier et al. 1998; Wandel et al. 1999). This corresponds to an Eddington luminosity $L_{\text {edd }} \simeq 9 \times 10^{44} \mathrm{erg} \mathrm{s}^{-1}$.

Our model gives us an estimate of the total luminosity emitted by the central engine in NGC 7469, from the UV to the hard $\mathrm{X}$-rays (if we admit that the UV emission is in large part dominated by the reprocessing of X-rays, it is equal to the downscattered emission emitted by the corona). Assuming a distance of $70 \mathrm{Mpc}$ (with $H_{0}=75 \mathrm{~km} \mathrm{~s}^{-1} \mathrm{Mpc}^{-1}$ ), the luminosity varies between 2 and $3 \times 10^{44} \mathrm{erg} \mathrm{s}^{-1}$ i.e. of the order of $L_{\text {edd }}$, meaning that the source accretes near its Eddington rate.

It is known that for such high accretion rate, the inner part of the accretion disc, which is dominated by the radiation pressure, is viscously and thermally unstable (Lightman \& Eardley 1974; Shakura \& Sunyaev 1976) and may be subject to violent clumping instabilities (Begelman 2001; Turner et al. 2002). If the disc remains geometrically thin, most of the disc mass form very dense clumps embedded in a tenuous hot corona. In this case however, we expect very soft spectra unless the covering fraction of the cold clouds is small which results however in low reflection component (Malzac \& Celotti 2002). This does not agree with the value of $\Gamma$ and $R$ observed in NGC 7469 .

A possible solution could be the presence of disc-corona configurations where a major fraction of the accretion power is released in the corona, as those proposed by Svensson \& Zdziarski (1994), Merloni \& Fabian (2002), and more recently by Merloni (2003). The presence of such strong coronae indeed prevents the development of disc instabilities. Merloni (2003) found for example new thermally and viscously stable optically thick accretion disc solutions for magnetized turbulent flows. Assuming that the magnetic field amplification, via MRI, is balanced by buoyant escape, they obtained high viscosity solutions where a large fraction of the accretion power is liberated in the corona. These solutions appear only above a critical accretion rate of the order of few tenths of the Eddington one, which corresponds to the case of NGC 7469. The disc geometry being conserved in these solutions, we also expect the reflection normalization to be of the order of unity as observed.

If this interpretation is correct, it is likely that parts of the disc involved in the corona formation process are

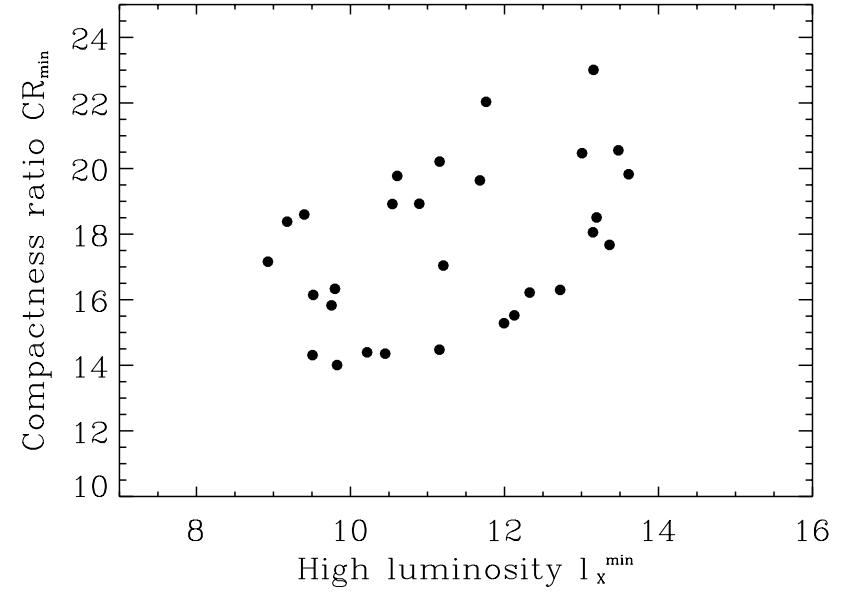

Fig. 6. The compactness ratio $C R_{\min }=\frac{l_{X}^{\min }}{l_{\mathrm{UV}}}$ versus the $\mathrm{X}$-ray compactness $l_{\mathrm{X}}^{\min }$ obtained assuming a 1 light day for the $\mathrm{X}$-ray region size (cf. Sect. 4.4 for details)

intrinsically weakly luminous, most of the energy being released mechanicly/magneticaly rather than radiatively. The observed UV flux would then be dominated by the reprocessed emission in agreement with the observations. Moreover, it could also explain why the black body temperature $k T_{\mathrm{bb}}$ deduced from our fits is significantly smaller than the inner temperature (at $\sim 10$ Schwarzschild radii) of $\sim 50 \mathrm{eV}$ expected for a standard accretion disc (i.e. without corona) assuming the black hole mass and accretion rate estimates of Collier et al. (1998) from optical data analysis.

\subsection{A pair free corona}

As already said, the code we use can give us the total X-ray and UV fluxes (and consequently the luminosity) emitted by the corona and the disc respectively. Now, from the variability in both bands, we can also estimate an upper limit of the size of the emitting regions, $R_{\mathrm{X}}$ and $R_{\mathrm{UV}}$, and consequently a lower limit on the compactnesses $l_{\mathrm{X}, \mathrm{UV}}=\frac{\sigma_{\mathrm{L}} \mathrm{L}_{\mathrm{X}, \mathrm{UV}}}{m_{\mathrm{e}}{ }^{3} R_{\mathrm{U}, \mathrm{UV}}}$.

The X-rays appears to be variable on a timescale of less than 1 day (cf. N98, N00) thus $l_{\mathrm{X}}>l_{\mathrm{X}}^{\min }=\frac{\sigma_{1} L_{\mathrm{X}}}{R_{1} m_{\mathrm{e}} c^{3}}$ where $R_{1}$ is a 1 light day length. On the other hand the UV vary on a longer time scale of the order of 5 days (Wanders et al. 1997; N98; N00; Collier \& Peterson 2001). Then the compactness ratio $C R=\frac{l_{\mathrm{X}}}{l_{\mathrm{UV}}}>C R_{\min }=\frac{l_{\mathrm{X}}^{\min }}{l_{\mathrm{UV}}} \simeq 5 \frac{L_{\mathrm{X}}}{L_{\mathrm{UV}}}$. We have reported in Fig. 6 , the $\left(C R_{\min }, l_{\mathrm{X}}^{\mathrm{min}}\right)$ values corresponding to our 29 daily observations. We obtained a slight correlation between these 2 variables. We also note that they only vary by about $50-60 \%$ during the campaign.

These results clearly disagree with the pair-dominated expectations computed by Ghisellini \& Haardt (1994). Their Fig. 2 shows that, if the corona of NGC 7469 is pair dominated, its compactness ratio has to decrease and its high compactness to increase (both by a factor $\sim 10$ ) from the softest state $\left(\Gamma=2.01, k T_{\mathrm{e}}=266 \mathrm{keV}\right)$ to the hardest state $(\Gamma=1.7$, $k T_{\mathrm{e}}=420 \mathrm{keV}$ ) reach by the source during the 29 days of observation. It would thus require very large changes (of factor 5 or more) of the characteristic size of both the UV and X-ray 
emitting regions for our results to be compatible with the pair dominated case. This is in contradiction with the observations. For instance, the observed UV flux varying by only 50-60\% imposes the size of the emitting region (assumed to be optically thick) to vary at most by the same amount.

\section{Conclusion}

We have re-analyzed in this paper the simultaneous 30 days IUE/XTE observation of NGC 7469 done in 1996, adopting and fitting directly to the data a detailed model of the Comptonized spectrum. This contrasts with previous spectral analysis where a simple power law was used to model the continuum. We were thus able to fit simultaneously the data from the UV to the hard X-ray band in a self-consistent way and to obtain direct constraints on the physical parameters of the disccorona system, like the temperature and optical depth of the corona.

The model we use is able to explain well the spectral shape of the different UV/X-ray spectra but also the UV and X-ray flux variability. The absence of a clear correlation between the UV and X-ray fluxes, as reported by N98, was thus clearly misleading. It underlines the importance of the use of consistent models when fitting so intimately related energy bands. In this context, the observed delay of $\sim 0.14$ day between the UV and X-ray light curves of NGC 4051, as reported by Mason et al. (2002), may not correspond to a response delay between the UV and $\mathrm{X}$-ray emitting region.

Concerning NGC 7469, the data appear to be roughly consistent with a slab geometry where all the power is liberated inside the corona and where the UV flux is dominated by the reprocessing. We note however that more photon-starved geometries also agree with the data.

We also find very interesting correlations between the different model parameters as the one between the corona temperature and the UV flux. If it can be easily explained in the framework of thermal comptonization model (an increase of the UV flux means an increase of the corona cooling and thus, in general, a decrease of the corona temperature), it necessarely implies slight changes of the disc-corona configuration during the 29 days of observation. We thus propose a model where the main origin of the variability is a change of the global corona geometry. The more patchy it becomes, the hotter the temperature. In the same time, the reprocessed flux, i.e. the UV emission, decreases.

We also found an interesting correlation between the corona optical depth and the X-ray flux which is, a priori, not expected by Comptonization models and may result from a different, but connected, process linked to the corona formation.

We show that NGC 7469 is apparently accreting at nearly the Eddington rate. The presence of a reflection component of the order of unity and of a relatively hard spectrum suggest the presence of a magnetically dominated disc-corona system in this source where most of the accreting energy is released in the corona. Such solutions have recently been shown to be thermally and viscously stable by Merloni (2003). We finally found strong evidences in favor of a pair free corona.
We note finally that the lack of precise data above $20 \mathrm{keV}$ prevents however any detailed spectral study of the soft $\gamma$ emission of the source. For instance, the study of the variability of the high energy cut-off, would have given additionnal constraints on the model parameters, especially on the corona temperature. Such very broad band study would require simultaneous observations with high energy satellites like XMM and INTEGRAL.

\section{References}

Barr, P. 1986, MNRAS, 223, 29P

Begelman, M. C. 2001, ApJ, 551, 897

Berkley, A. J., Kazanas, D., \& Ozik, J. 2000, ApJ, 535, 712

Brandt, W. N., Fabian, A. C., Nandra, K., \& Tsuruta, S. 1993, MNRAS, 265, 996

Chiang, J., Reynolds, C. S., Blaes, O. M., et al. 2000, ApJ, 528, 292

Chiang, J. 2002, ApJ, 572, 79

Clavel, J., Nandra, K., Makino, F., et al. 1992, ApJ, 393, 113

Collier, S. J., Horne, K., Kaspi, S., et al. 1998, ApJ, 500, 162

Collier, S., \& Peterson, B. M. 2001, ApJ, 555, 775

De Rosa, A., Fabian, A. C., \& Piro, L. 2002, MNRAS, 334, L21

Done, C., Ghisellini, G., \& Fabian, A. C. 1990, MNRAS, 245, 1

Edelson, R. A., Alexander, T., Crenshaw, D. M., et al. 1996, ApJ, 470, 364

Edelson, R., Koratkar, A., Nandra, K., et al. 2000, ApJ, 534, 180

Edelson, R., Turner, T. J., Pounds, K., et al. 2002, ApJ, 568, 610

Ghisellini, G., Haardt, F., \& Matt, G. 1994, ApJ, 429, L53

Gondek, D., Zdziarski, A. A., Johnson, W. N., et al. 1996, MNRAS, 282,646

Guainazzi, M., Matsuoka, M., Piro, L., Mihara, T., \& Yamauchi, M. 1994, ApJ, 436, L35

Haardt, F. 1993, ApJ, 413, 680

Haardt, F., \& Maraschi, L. 1991, ApJ, 380, L51

Haardt, F., \& Maraschi, L. 1993, ApJ, 413, 507

Haardt, F. 1994, Ph.D. dissertation, SISSA, Trieste (H94)

Haardt, F., Maraschi, L., \& Ghisellini, G. 1997, ApJ, 476, 620

Henri, G., \& Pelletier, G. 1991, ApJ, 383, L7

Jourdain, E., Bassani, L., Bouchet, L., et al. 1992, A\&A, 256, L38

Kriss, G. A., Peterson, B. M., Crenshaw, D. M., \& Zheng, W. 2000, ApJ, 535, 58

Lightman, A. P., \& Eardley, D. M. 1974, ApJ, 187, L1

Lightman, A. P., \& White, T. R. 1988, ApJ, 335, 57

Liu, B. F., Mineshige, S., \& Shibata, K. 2002, ApJ, 572, L173

Maisack, M., Johnson, W. N., Kinzer, R. L., et al. 1993, ApJ, 407, L61

Malkan, M. A., \& Sargent, W. L. W. 1982, ApJ, 254, 22

Malzac, J., \& Celotti, A. 2002, MNRAS, 335, 23

Malzac, J., \& Jourdain, E. 2000, A\&A, 359, 843

Malzac, J., \& Petrucci, P. 2002, MNRAS, 336, 1209

Malzac, J., Beloborodov, A. M., \& Poutanen, J. 2001, MNRAS, 326, 417

Malzac, J., Mouchet, M., \& Dumont, A. M. 2002, A\&A, submitted

Maoz, D., Edelson, R., \& Nandra, K. 2000, AJ, 119, 119

Maoz, D., Markowitz, A., Edelson, R., \& Nandra, K. 2002, AJ, 124 1988

Marshall, H. L., Carone, T. E., Peterson, B. M., et al. 1997, ApJ, 479, 222

Mason, K. O., McHardy, I. M., Page, M. J., et al. 2002, ApJ, 580, L117

Matt G., 2001, in X-Ray Astronomy, Stellar Endpoints, AGN, and the Diffuse X-ray Background, Bologna, Italy 1999, ed. N. E. White, G. Malaguti, \& G. G. C. Palumbo, AIP Conf. Proc. 599, 209 [astro-ph/0007105] 
Merloni, A. MNRAS, 341, 1051

Merloni, A., \& Fabian, A. C. 2002, MNRAS, 332, 165

Meyer, F., \& Meyer-Hofmeister, E. 1994, A\&A, 288, 175

Meyer, F., Liu, B. F., \& Meyer-Hofmeister, E. 2000, A\&A, 361, 175

Nandra, K., Clavel, J., Edelson, R. A., et al. 1998, ApJ, 505, 594 (N98)

Nandra, K., Le, T., George, I. M., et al. 2000, ApJ, 544, 734 (N00)

Nandra, K., \& Papadakis, I. E. 2001, ApJ, 554, 710

Nayakshin, S., Kazanas, D., \& Kallman, T. R. 2000, ApJ, 537, 833

Papadakis, I. E., Nandra, K., \& Kazanas, D. 2001, ApJ, 554, L133

Perola, G. C., Matt, G., Cappi, M., et al. 2002, A\&A, 389, 802

Petrucci, P. O., Haardt, F. Maraschi, L., et al. 2000, ApJ, 540, 131

Petrucci, P. O., Henri, G., \& Pelletier, G. 2001, A\&A, 374, 719

Petrucci, P. O., Haardt, F., Maraschi, L., et al. 2001, ApJ, 556, 716

Różańska, A., \& Czerny, B. 1996, Acta Astron., 46, 233

Różańska, A., \& Czerny, B. 2000, A\&A, 360, 1170

Shakura, N. I., \& Suniaev, R. A. 1976, MNRAS, 175, 613

Shemmer, O., Romano, P., Bertram, R., et al. 2001, ApJ, 561, 162
Shields, G. A. 1978, Nature, 272, 706

Stern, B. E., Poutanen, J., Svensson, R. , Sikora, M., \& Begelman, M. C. 1995, ApJ, 449, L13

Svensson, R. 1996, A\&AS, 120, C475

Svensson, R., \& Zdziarski, A. A. 1994, ApJ, 436, 599

Turner, N. J., Stone, J. M., \& Sano, T. 2002, ApJ, 566, 148

Turner, T. J., Weaver, K. A., Mushotzky, R. F., Holt, S. S., \& Madejski, G. M. 1991, ApJ, 381, 85

Turner, T. J., George, I. M., \& Mushotzky, R. F. 1993, ApJ, 412, 72

Uttley, P., McHardy, I. M., Papadakis, I. E., Cagnoni, I., \& Fruscione, A. 2000, MNRAS, 312, 880

Wandel, A., Peterson, B. M., \& Malkan, M. A. 1999, ApJ, 526, 579

Wanders, I., Peterson, B. M., Alloin, D., et al. 1997, ApJS, 113, 69

Welsh, W. F. 1999, PASP, 111, 1347

White, T. R., Lightman, A. P., \& Zdiziarski, A. A. 1988, ApJ, 331, 939

Zdziarski, A. A., Zycki, P. T., \& Krolik, J. H. 1993, ApJ, 414, L81

Zdziarski, A. A., \& Grandi, P. 2001, ApJ, 551, 186 